\title{
Relaciones generacionales: Entre la violencia y el afecto.
}

\section{Generational relations: Between violence and affection.}

Fredy Aguilar Rodríguez

Universidad Técnica de Ambato, Ecuador

María Gabriela de la Cruz

Universidad Internacional del Ecuador, Ecuador

Ítalo Chicaiza Aucapiña

Investigador independiente, Ecuador

Autor para correspondencia: wf.aguilar@uta.edu.ec; madelacruzla@uide.edu.ec; italochicaiza2012@hotmail.es

Fecha de recepción: 15 de noviembre del 2018 - Fecha de aceptación: 1 de abril del 2019

Resumen: El presente trabajo tiene como fin provocar una reflexión profunda sobre las relaciones afectivas entre adultos y niños, para determinar su grado de incidencia en el crecimiento y desarrollo saludable de jóvenes, niños y niñas. Para alcanzar este objetivo se extraen cifras de la línea base realizada en la provincia del Napo, corroborada y comparada con otros trabajos investigativos de la localidad y a nivel nacional; seguidamente se presentan los resultados obtenidos en la encuesta cantonal sobre el trato entre adultos y niños/as, desde el Concejo Cantonal de la Niñez y Adolescencia. Finalmente, se analizarán las denuncias de vulneración de derechos registradas por la Junta Cantonal de Protección de Derechos para intentar desembocar en una especie de bosquejo hacia la construcción de una cultura de paz. La complejidad del tema provoca un abordaje interdisciplinario, más allá de las estadísticas y concepciones hay que tener una mirada plural y amplia que ahuyente las tendencias antagónicas que consideran sin valor alguno el tema afectivo, y sentencian invariables los patrones de relación socio-afectivas.

Palabras claves: socialización; jóvenes; infancias; vulneración; violencias

\begin{abstract}
The present dissertation has as purpose to promote a deep reflection on the affective relations between adults and children, to determine its incidence degree in the growth and healthy development of young people, boys and girls. To achieve this objective, figures are extracted from the baseline conducted in the province of Napo, corroborated and compared with other research work in the local and national level; once results were obtained in the cantonal survey on the treatment of adults and children they are presented, from the Cantonal Council of Children and Adolescents. Finally, complaints of infringement of rights registered by the Cantonal Protection of Rights Board will be analyzed to try to lead to a kind of outline towards the construction of a culture of peace. The complexity of the subject promotes an interdisciplinary approach, beyond the statistics and conceptions we must have a plural and wide view that scare away the antagonistic tendencies that consider the affective issue without value, and the invariable adjudication of the socio-affective relationship patterns.
\end{abstract}

Key Words: socialization; youth; childhood; vulnerability; violence 


\section{Introducción}

Según los estudios de infancias y juventudes (Sánchez, 1989; 2004) los niños y niñas fueron invisibilizados y tratados como adultos en miniatura, estableciendo relaciones unidireccionales, donde los sueños, las ideas, las palabras y acciones de niños y niñas no eran tomados en cuenta, y casi siempre se convertían en motivación para la aplicación de castigos. Pero esta historia aún la vivimos hoy en día, de diferentes maneras e intensidad.

En la actualidad es muy usual el término "derechos", a tal punto que muchas personas tienden a confundir su significado. Más aún, cuando se trata de derechos de niños, niñas y jóvenes. Más allá del concepto clásico, ser titular de derechos implica acceder a los servicios elementales que cubran las necesidades básicas, posibiliten los caminos para alcanzar las metas soñadas y desarrollar al máximo habilidades y destrezas.

El obstáculo para que una persona alcance su realización y sea titular de derechos, es la violencia con sus diferentes manifestaciones que se interiorizan a tempranas edades (Aguilar y Tingo, 2016). Aunque todos compartimos ciertos patrones comunes, el ambiente, la cultura y otros factores que determinan nuestro crecimiento y desarrollo. Las relaciones socio-afectivas "saludables" entretejidas en el trayecto vital contribuirán a generar espacios verdaderamente democráticos. A continuación, presentamos el esfuerzo por entender las formas de relación adulto céntricas, en las que muchos niños, niñas y jóvenes se encuentran.

Breve descripción de categorías:

En el Art. 35 de la Constitución del Ecuador aprobada en el 2008, dice: "Las personas adultas mayores, niñas, niños y adolescentes, mujeres embarazadas, personas con discapacidad, personas privadas de libertad y quienes adolezcan de enfermedades catastróficas o de alta complejidad, recibirán atención prioritaria y especializada en los ámbitos público y privado".

La Organización Mundial de la Salud, afirma que la violencia es el uso intencional de la fuerza o el poder físico, de hecho, o como amenaza, contra uno mismo, otra persona, un grupo o comunidad, que cause o tenga muchas probabilidades de causar lesiones, muerte, daños psicológicos, trastornos del desarrollo o privaciones.

Para Ferrándiz la violencia es entendida como una relación política y de poder asimétrica (Ferrándiz, 2004). Esta puede agruparse en: política, estructural, simbólica y cotidiana. La violencia política se interrelaciona estrechamente con la violencia directa o intencional, y es administrada en el nombre de una ideología política. La violencia estructural se determina por la organización política-económica de las sociedades que imponen determinadas condiciones de sufrimiento físico y emocional a los sectores más vulnerables (Bourgois, 2002). La violencia simbólica, en términos de Bourdieu (2000) constituye una dominación que opera en un nivel íntimo de las personas debido a un reconocimiento-desconocimiento de las estructuras de poder, de manera especial, por los dominados que tienden a cooperan con su propia dominación. Por último, la violencia cotidiana caracterizada por los conflictos íntimos en determinados contextos (Bourgois, 2010). 
Para nuestro caso es importante describir la cultura Kichwa, entendida esta como el modo de vida de una población, es decir, el conjunto de símbolos, códigos y redes de significado por los cuales las instituciones toman un sentido para los agentes y se encarnan en conductas más o menos codificadas (Bourdieu, 2005). El término kichwa hace referencia al nombre étnico que reciben los pobladores que habitan gran parte de la Provincia de Napo (Böll, 1997).

\section{Precisiones metodológicas}

Siguiendo el proceso científico, el abordaje reflexivo de este tema se sustenta en la observación de los hechos socio-culturales (Aguilar, 2018), en términos metodológicos la investigación cuantitativa fue primordial porque permitió explicar los vínculos entre variables como violencia y afectividad, contextos étnicos y reproducción social. Luego están el análisis y síntesis de la información recabada en anteriores trabajos investigativos, y sobre todo en el trabajo realizado por organismos de protección de derechos en esta localidad, en especial la encuesta cantonal sobre las relaciones afectivas entre adultos y niños.

La encuesta se aplicó a una muestra representativa de 1000 niños, niñas y adolescentes entre 10 y 14 años, tomando en cuenta que están en la etapa escolar y que sus características de crecimiento y desarrollo brindan una mejor oportunidad para recoger sus apreciaciones en cuanto a la relación con sus padres.

Tomando como referencia desde la sociología el esquema de Berthelot (2003), se puede decir que este trabajo analítico sigue el siguiente camino: Esquema actancial: según el cual todo fenómeno estudiado es el resultado del comportamiento de los actores implicado; esquema "hermeneutique": un fenómeno o comportamiento es la expresión de un sentido que hay que actualiza; esquema "dialectique": donde la realidad está atravesada de contradicciones que deben ser superadas de manera que está siempre en construcción (Auyero, 2013).

Básicamente se ha realizado un recorrido analítico de la información contenida en los siguientes trabajos investigativos:

- Línea base del cumplimiento de derechos en el Cantón Archidona, hecha en el 2009;

- Investigación: Factores contribuyentes a la desnutrición en niños entre 6 meses y 5 años en el Cantón Archidona (2011).

- Investigación: El aporte de los cotopaxenses al desarrollo socio-cultural de Archidona.

- Encuesta Nacional del ODNA: niñez y su situación a inicios del siglo XXI.

- Encuesta Cantonal: Formas de relación entre adultos y niños/as en el Cantón Archidona (2012).

- Informe de Derechos Humanos 2009-2013, del Programa Andino de Derechos Humanos de la Universidad Andina;

- Informe de rendición de cuentas de la Junta Cantonal de Protección de Derechos del Cantón Archidona (2011-2015). 


\section{Resultados}

En el año 2003, luego de varios años de lucha permanente de varios organismos, colectivos y personas que exigían el cumplimiento de los mandatos de la Convención de los Derechos del Niño, el Ecuador aprobó y publicó el Código de la Niñez y Adolescencia. Este hecho, sin duda marca un nuevo camino con matices particulares y retos a largo alcance, ya que el libro tercero de este cuerpo legal, ordena crear un Sistema Nacional Descentralizado de Protección Integral de Niñez y Adolescencia.

Anteriormente, en el año de 1998, se aprobó la penúltima Constitución, en la que se reconoce al Ecuador como un Estado Plurinacional y Multiétnico. Legalmente es un hecho trascendental, pero en la práctica los derechos de los pueblos y nacionalidades y particularmente de los niños y niñas de este importante sector poblacional, siguen siendo violentados debido a múltiples factores.

Teniendo en cuenta los dos hechos históricos, arriba mencionados, lentamente en el país se empieza a construir el Sistema Nacional Descentralizado de Protección Integral de Niñez y Adolescencia (SNDPINA). Archidona fue el segundo cantón en la Provincia de Napo en crear, mediante Ordenanza Municipal, a finales del año 2005 e inicios del 2006, el Concejo Cantonal de Niñez y Adolescencia, pero recién en el año 2010 se eligió al responsable administrativo o Secretario Ejecutivo. A partir de este hecho se han realizado varios intentos por socializar y sensibilizar a la población local (educadores, autoridades y organizaciones kichuas) sobre la ampliación de los derechos de todos.

En el año 2009, el Consejo Nacional de la Niñez y Adolescencia junto a UNICEF y el Observatorio de los Derechos de la Niñez y Adolescencia, levantaron una línea base en Archidona y Tena, teniendo como soporte técnico el índice de cumplimiento de derechos de la Niñez y -adolescencia - IDN, la misma que es una medida sintética que permite monitorear el nivel de cumplimiento de derechos de manera objetiva e integral. Los resultados arrojados por este trabajo en mención son válidos indistintamente para los dos cantones amazónicos.

Este índice está conformado por varios indicadores que dan cuenta de la garantía de derechos de acuerdo al ciclo de vida: IDN1 Primeros Años (0 a 5 años), IND2 Edad Escolar (6 a 11 años) e IDN3 Adolescentes (12 a 17 años). Cada uno de ellos abarca tres derechos fundamentales, representados por un indicador social. La combinación de los tres indicadores se transforma en una calificación con una escala que va de 0 (deficiente) a 10 (sobresaliente). Por lo tanto, el desafío que se presenta tanto para el gobierno cantonal de Archidona como para sus habitantes es alcanzar la calificación de 10 puntos para así asegurar el pleno cumplimiento de los derechos de la niñez y la adolescencia (Encuesta cantonal de niñez y adolescencia de Archidona, agosto 2009, p. 6). 


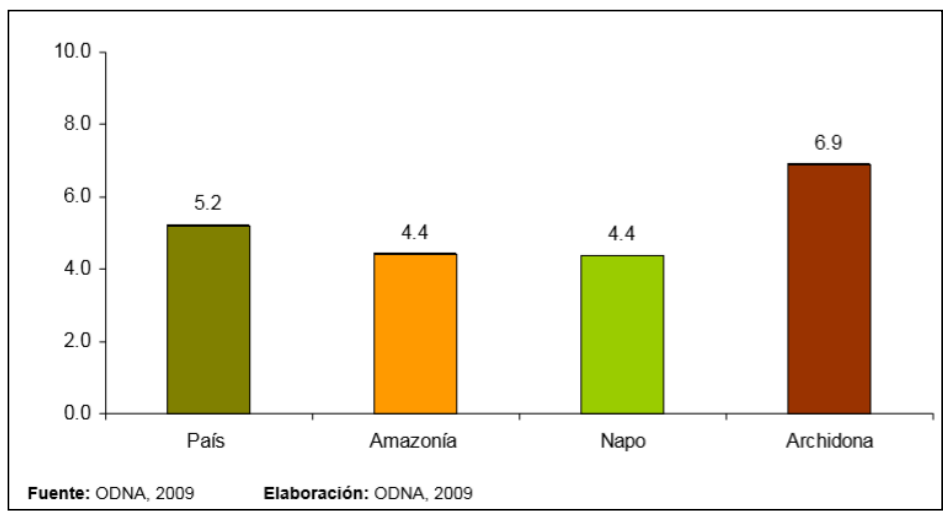

Llama la atención la calificación de 6.9/10 que el Cantón Archidona obtiene a nivel nacional, sabiendo que la nota mínima es 7/10 para demostrar que en este cantón si se cumplen los derechos de niñez y adolescencia y "pasar el año" sin arrastre.

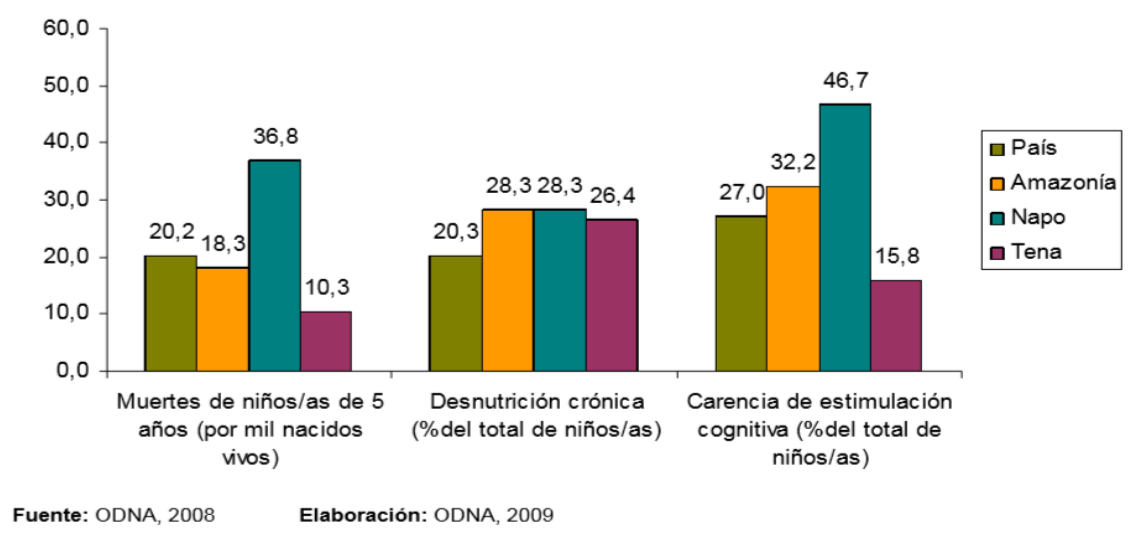

Estos resultados no coinciden con los obtenidos en el año 2011, donde se enfatizó los factores contribuyentes de la desnutrición de niños entre 6 y 5 años de edad del cantón. Aquí se dice que en el Ecuador se calcula que el 33.9\% de niños menores de 5 años sufren de la desnutrición, en el cantón Archidona, la tasa es 35.9\%.

Vale resaltar que, en el trabajo investigativo primero, se toma en cuenta a niños de 0 a 5 años, en cambio en el 2011 la población referida es el universo de niñez, es decir, de 0 a 12 años de edad. Pese a esta particularidad el dato es alarmante, tomando en cuenta los esfuerzos del Gobierno central y los órganos locales responsables de la salud. 


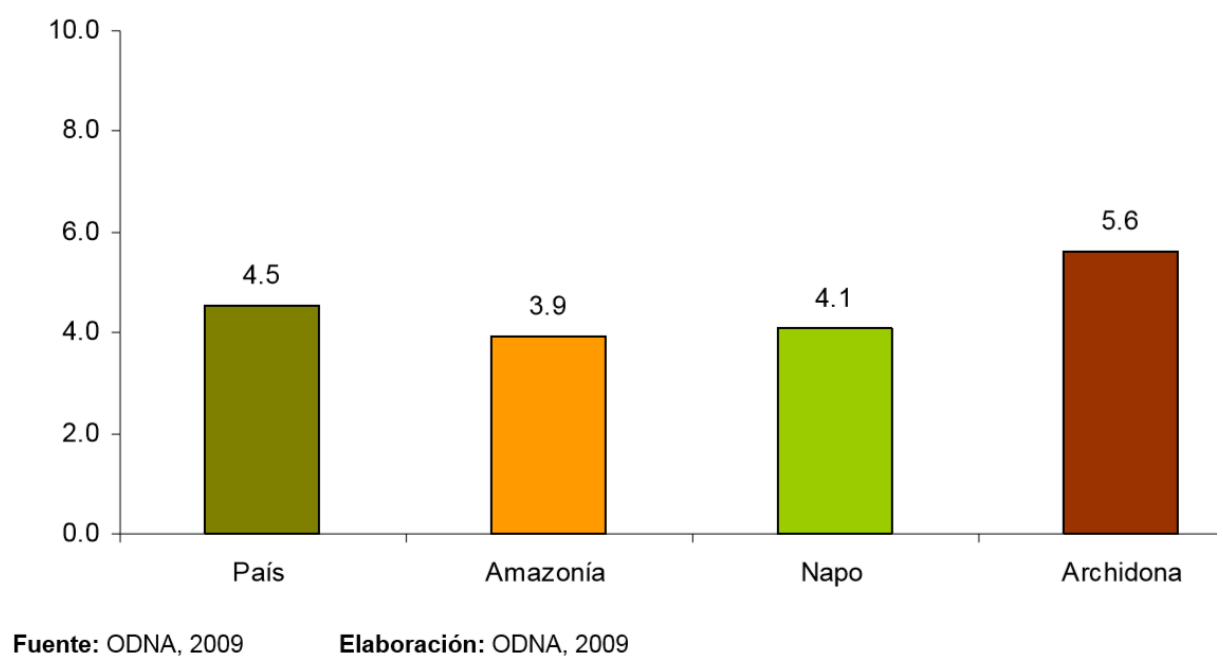

En el 2009, el indicador 2 que se refiere al cumplimiento de tres garantías básicas de los niños y niñas de 6 a 11 años: al desarrollo intelectual, a vivir sanamente libre de miedo y amenazas y a jugar y compartir con los padres, la nota es 5.6 sobre 10. Esta calificación, se debe a que en el Cantón el 55.9\% de niños y adolescentes entrevistados afirman que sus padres resuelven los problemas con el castigo físico y no con el diálogo.

\section{Sobre la encuesta cantonal}

En la encuesta levantada, obtuvimos los siguientes resultados, enfatizando la relación entre adultos y niños, niñas y adolescentes de 9 a 13 años de edad. Pero, además, paralelamente se aplicó la encuesta a los padres de familia, con el fin de apreciar mejor la visión de los adultos a la hora de educar y criar a sus hijos. Semelín (1983) afirma:

Los malos tratos, los abusos, la obligación al trabajo y la escasa consideración de los derechos de niños, niñas y adolescentes son visualizados como parte de un conjunto de creencias y mitos que fortalecen la autoridad de los padres. Además, la no consideración de crianza y educación como cuestiones de interés público alimenta una ideología que no pone restricciones a los comportamientos y derechos supuestos de los padres sobre los hijos e hijas, y permiten la apropiación del cuerpo y de los comportamientos de niños y niñas (Semelín, 1983, p. 14).

Al pedirles que califiquen la relación con sus padres, el $61 \%$ afirmó que es muy buena, en cambio al preguntarles a sus padres, constatamos que tan solo el $24 \%$ dijo que es muy buena. ¿A qué se debe esta gran diferencia? Tomando en cuenta que el $80 \%$ de la población de Archidona es Kichwa, y el resto lo conforman "colonos" de varios sectores de la sierra y otros lugares del mundo, ya podemos barajar algunas pistas de reflexión. 


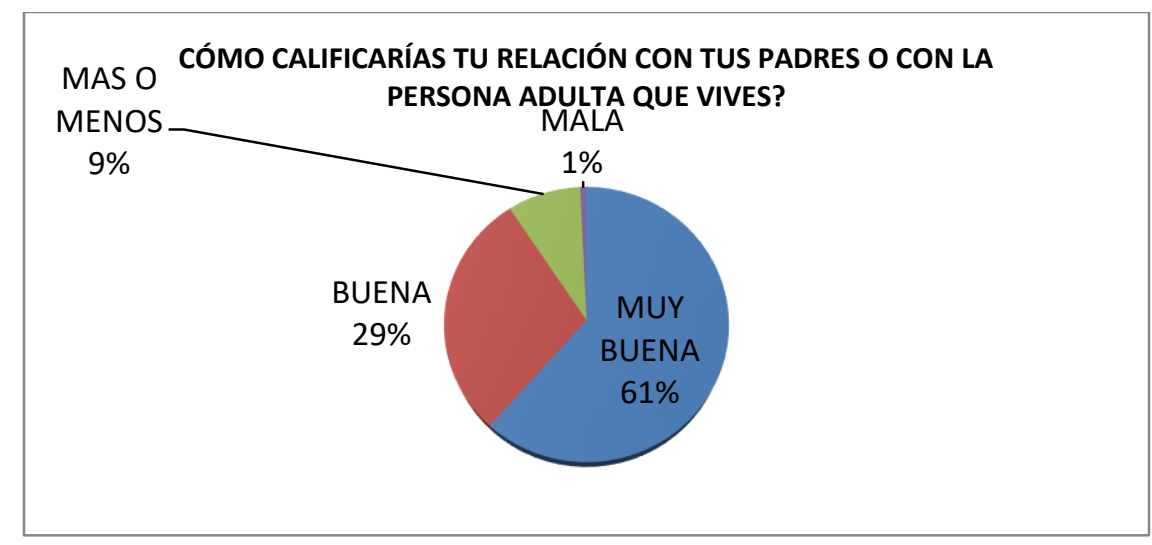

Fuente: elaboración autores.

Durante la encuesta percibimos que los padres del sector urbano respondieron en su mayoría que su relación es buena, porque últimamente están teniendo más conflictos y ya no les pueden castigar ni corregir porque les responden que les van a denunciar. En cambio, en el sector rural, la mayoría de padres dijo que su relación es muy buena y mencionaron que sus hijos son más "obedientes".

En la respuesta "más o menos", la diferencia entre hijos y padres es de 3 puntos y en la respuesta "mala" coinciden los dos grupos poblacionales con el 1\%. La diferencia, puede darse debido a la ambigüedad que conlleva el término más o menos, ya que en el uso popular es muy común escucharlo, pese a que las circunstancias sean buenas, pero es usual, para no ir a los extremos de las situaciones e incluso de los estados emotivos.

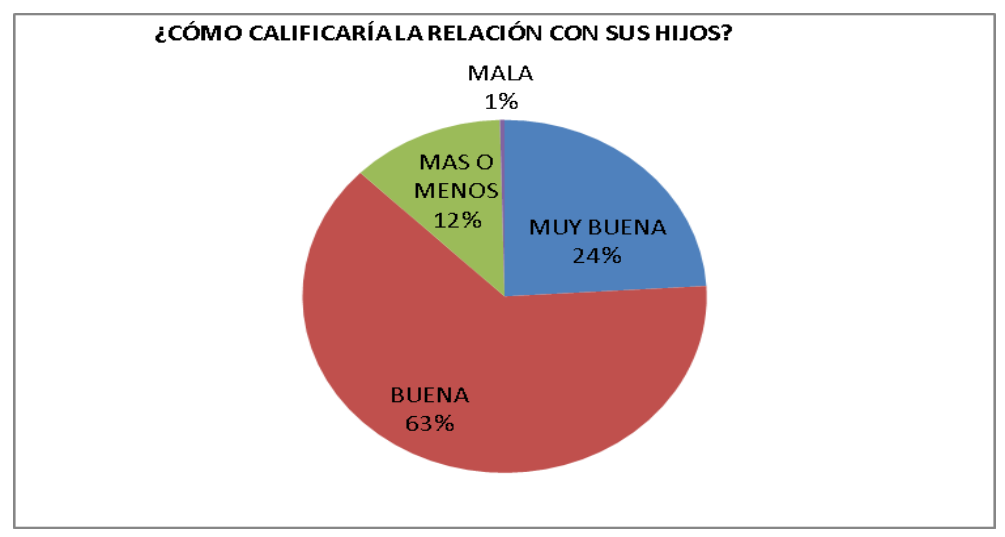

Fuente: elaboración autores.

La población objetivo está conformado por hombres y mujeres indistintamente, pero los las niñas y adolescentes mujeres las que califican de muy buena la relación con sus padres (papá y mamá), en cambio los padres-varones son los que en su mayoría dijeron que la relación con sus hijos es buena.

Algunos autores como Palacios, J., Marchesi, A., y Coll, C. (2002), sostienen que después de los 6 años de edad hay un aumento global de "conductas agresivas ", aunque dado el aumento paralelo de conductas sociales positivas, la proporción de las agresivas frente a las 
amistosas haría ver un decremento de aquellas con la edad. Otros (Sánchez, 2007; Del Olmo, 2000) afirman que la agresión y la violencia responde a condiciones sociales, en especial a condiciones estructurales de vida. Este aumento caracterizaría sólo a los varones, los chicos más tendentes a manifestar confrontación abierta y las chicas más proclives a formas de agresión más sutiles, como la de promover el ostracismo, la difamación y la burla centrada en los atributos negativos de otros (Calle, 2012).

\section{Razones para dar o recibir castigos}

A continuación, se preguntó sobre las razones por las que los padres castigan a sus hijos y el $24 \%$ afirma que es por llegar tarde, el $23 \%$ por no hacer tareas en la casa y el $22 \%$ por obtener malas notas.

¿Qué significa llegar tarde? Deducimos que no es precisamente que los hijos se atrasan o incumplen los horarios, sino que no obedecieron y por lo mismo no llegaron a la hora que sus padres les dijeron que lleguen. La imposición siempre genera una contra respuesta o al menos malestar y rechazo, que puede ser expresado de diversas formas y conductas.

Observamos que hacer tareas en la casa, es cumplir con un trabajo que al mismo tiempo es variado y desproporcional, produciendo cansancio, evasión e incumplimiento. Finalmente, las "malas notas" son objeto de castigo porque no satisface las expectativas de sus padres, es decir, no aceptan que las notas, que son valores cuantitativos, no siempre toman en cuenta las diversas habilidades y competencias que los niños, niñas y adolescentes poseen. El espacio familiar y escolar se convierte en espacios de producción de violencias.

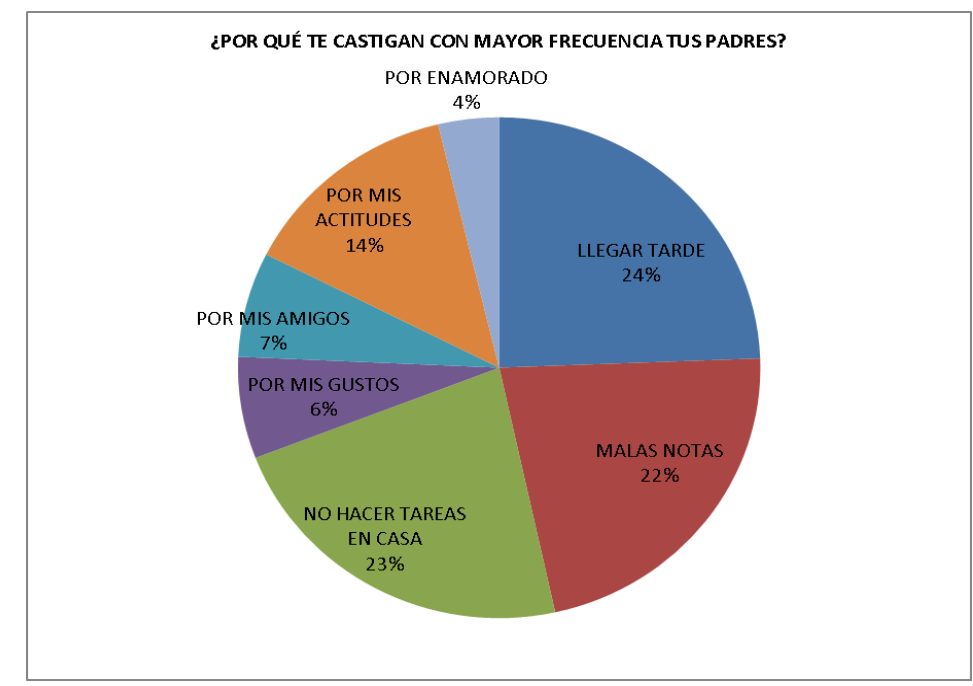

Fuente: elaboración autores.

Durante los años 2000, 2004 y 2010, el ODNA ejecutó a nivel nacional las encuestas y planteó la pregunta: ¿por qué te castigan con mayor frecuencia tus padres? El resultado fue similar al expuesto anteriormente, con la variación que la violencia no solo se reproduce, sino 
también se produce, por tanto, las instituciones son productoras y reproductoras de violencia cotidiana.

\section{Castigos más frecuentes}

El abordaje a los niños, niñas y adolescentes para entrevistarles sobre las reacciones de los padres a sus faltas y desobediencia, arrojó que las más recurrentes son: hablar y regañar (45\%), pegar (13\%), privar de gustos (9\%), poner ají (8\%) y ortigar (6\%). No resultan novedosas estas formas de relación y “costumbres" entre adultos y niños/as y adolescentes, al contrario, parece algo natural y pedagógicamente recomendable, reorientar las conductas mediante castigos de diversa índole.

La historia es la mejor maestra para decirnos que estas formas de relación responden a una lógica patriarcal, jerárquica y unilateral. Generalmente son “órdenes” impartidas por adultos que deben ser cumplidas por los "menores". Estos mandatos desembocan en conductas y sentimientos variados, pero los más evidentes en los niños son la ira, el miedo y la naturalización de estas prácticas, incluso, con la complicidad de los actores sociales (Bourdieu, 2000).

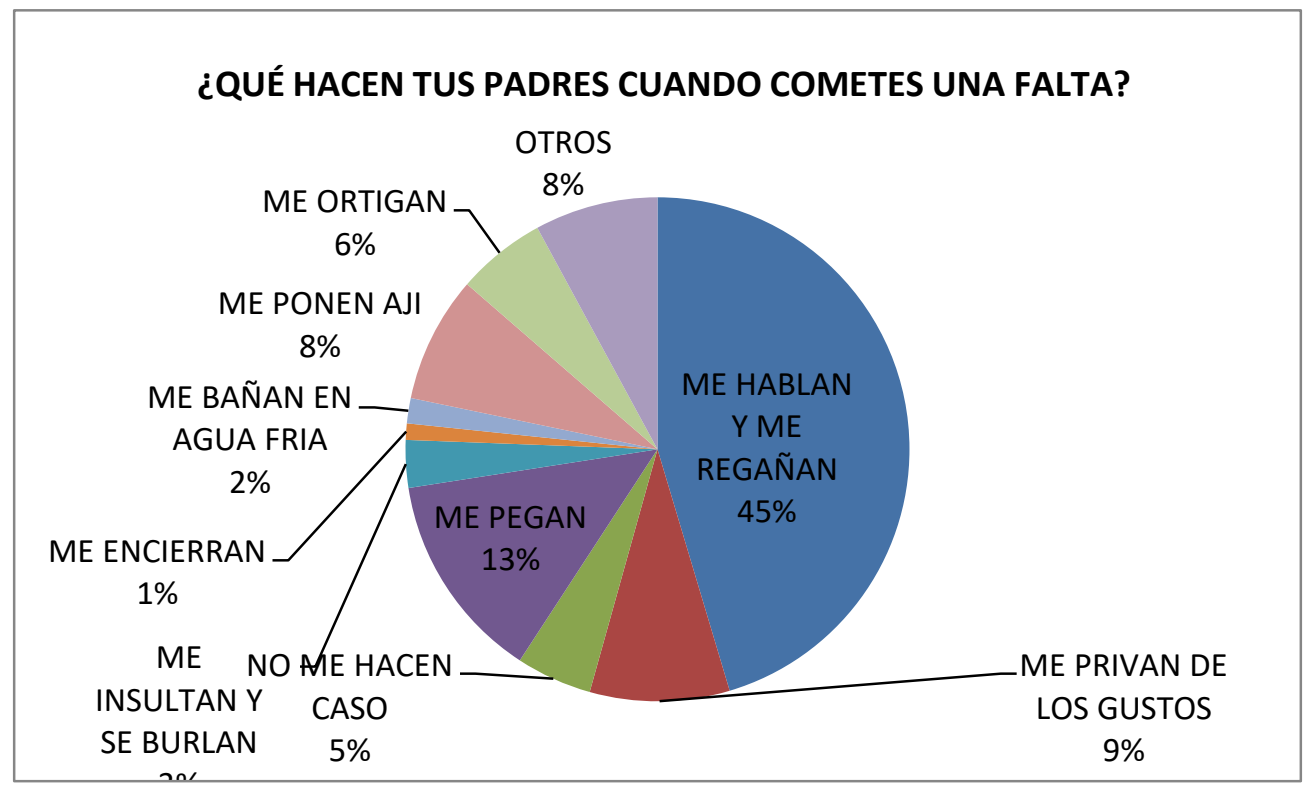

Fuente: elaboración autores.

Este gráfico muestra que, mientras los niños, niñas y adolescentes encuestados dicen que reciben más regaños y habladas (45\%), los padres dicen que no hacerles caso (42\%), hablan y regañan $(26 \%)$, pegar $(10 \%)$ y ponen ají y ortigan $(7 \%)$.

¿Contradicción o estrategia de circulación del poder? No hacerles caso, en muchos casos significa indiferencia, impotencia y desamparo total. Muchos padres entrevistados manifestaron que a partir de la aparición de los organismos que promocionan y defienden los derechos de niños, niñas y adolescentes, su autoridad se vio mermada y ya no pueden "hacer nada". Es decir, ya no pueden "ni tocarles". 
Casi todos los adultos entrevistados afirmar que, con el aparecimiento de las leyes a favor de los derechos de niñez y adolescencia, no solo su autoridad se vio restada, sino sus tradiciones y formas ancestrales de corregir a sus hijos cuando estos cometían faltas de diversa índole. Poner ají, por ejemplo, en las comunidades dichas de Archidona es muy común, porque según los abuelos, aparte de reorientar las conductas, hace a las personas más fuertes y tienen mejor visión cuando van de caza. El ají lo aplican en los ojos tanto de hombres y mujeres, pero las mujeres niñas son más humilladas cuando les aplican en su parte genital. No todo lo tradicional es popular ni todo lo popular merece ser conservado, tales como la pornografía, las drogas, el linchamiento, el maltrato a las mujeres y a los niños, producto de condiciones estructurales.

Desde la óptica legalista, muchas costumbres y formas de relación culturalmente aceptada, atentan contra los derechos de niños, niñas y adolescentes. Esta tesis resulta compleja dilucidarla porque las dinámicas culturales son cambiantes y tal como afirma Néstor García Canclini (2001), uno de los más respetables científicos sociales, es que debemos prestar mayor atención a los cambios más que a perenización de valores.

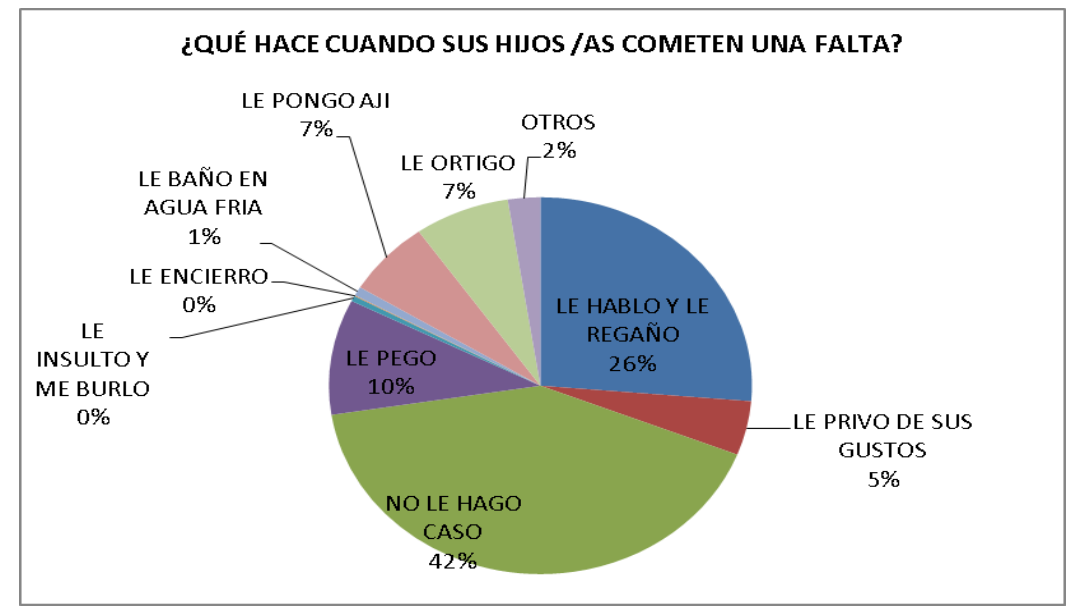

Fuente: elaboración autores.

La deficiente actuación del Estado en su afán de convertirse en garante de derechos es otro elemento que ha contribuido a generalizar la concepción anacrónica sobre los métodos de crianza que son determinados por las creencias de los padres.

Tipos de maltrato, según la Junta de Protección de Derechos.

Para contextualizar la alarmante vulneración de derechos de niños, niñas y adolescentes en el cantón Archidona, conviene anotar sintéticamente los casos denunciados en la Junta Cantonal de Protección de Derechos de Archidona ${ }^{1}$, desde el 2011 hasta el año 2015. Hechos registrados y clasificados por tipos de derechos vulnerados.

\footnotetext{
${ }^{1}$ Este organismo fue creado por el Gobierno Municipal de Archidona, a través del Concejo Cantonal de la Niñez y Adolescencia para dar cumplimiento al mandato legal del Código de la Niñez y Adolescencia. En el libro tecero, Art. 205 dice: Las Juntas Cantonales de Protección de Derechos son organismos de nivel operativo, con autonomía administrativa y funcional, que tienen como función pública la protección de los derechos individuales y colectivos de los niños, niñas y adolescentes, en el respectivo cantón. Las organizará cada municipalidad a nivel cantonal o
} 
Desde su creación, este organismo de protección de derechos, luego de receptar la denuncia y hacer el debido proceso, han dictado medidas que restablezcan los derechos vulnerados, pero los resultados, en muchas ocasiones no han sido los esperados, ya que una vez más se comprueba que esas medidas no responden a las necesidades y lógicas del Cantón y su población.

Veamos ilustrativamente los casos registrados sobre vulneración de derechos en base a los expedientes y denuncias atendidos por la Junta Cantonal de Protección de Derechos de la Niñez y Adolescencia de Archidona, para luego puntualizar algunas reflexiones en torno a las mismas.

Tabla. 1: Expedientes y denuncias 2011-2012

\begin{tabular}{ll}
\hline PERÍODO & $\begin{array}{l}\mathrm{N}^{\circ} \text { DE EXPEDIENTES Y } \\
\text { DENUNCIAS }\end{array}$ \\
\hline DE 01 DE MARZO DEL 2011 AL & 113 \\
28 DE DICIEMBRE DEL 2011 & \\
DE 01 DE ENERO DE 2012 AL 28 & 222 \\
DE DICIEMBRE DEL 2012 & \\
TOTAL & 335 \\
\hline
\end{tabular}

Fuente: elaboración autores.

Tabla. 2: Expedientes y denuncias enero - septiembre 2013

\begin{tabular}{cc}
\hline Período & $\begin{array}{c}\mathrm{N}^{\circ} \text { De Expedientes Y } \\
\text { Denuncias }\end{array}$ \\
\hline De 02 De Enero Del 2013 Al & 148 \\
10 De Septiembre Del 2013 & \\
Total De Casos Atendidos & 483 \\
\hline
\end{tabular}

Fuente: elaboración autores.

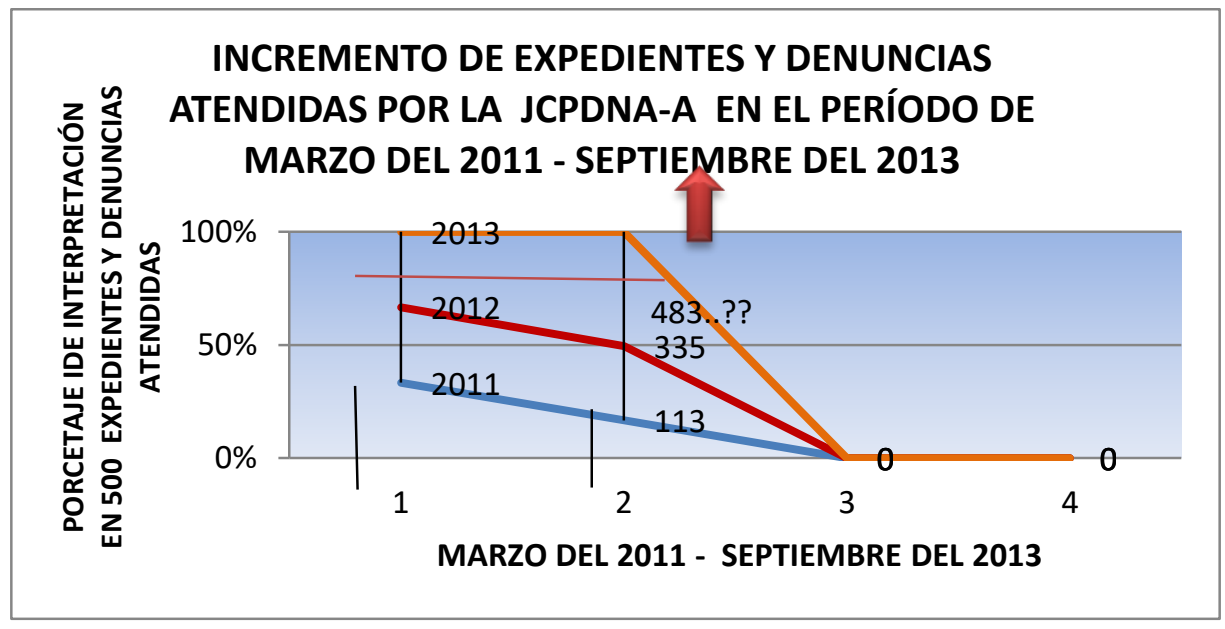

Fuente: elaboración autores. El gráfico muestra el crecimiento cada vez más ascendiente hasta el año 2013. Es cuestionable el número de casos que se habrán atendido hasta la actualidad.

parroquial, según sus planes de desarrollo social. Serán financiadas por el Municipio con los recursos establecidos en el presente Código y más leyes. 
A continuación, se presenta un detalle de casos de vulneración de derechos atendidos por la Junta Cantonal de Protección de Derechos de la Niñez y Adolescencia de Archidona:

Tabla. 3: Período de marzo del 2011 - diciembre del 2012 (durante los 2 años anteriores)

\begin{tabular}{ll}
\hline Tipos de maltrato & $\mathbf{N}^{\circ}$ denuncias \\
\hline Físico & 215 \\
Psicológico & 71 \\
Sexual & 15 \\
Discriminación & 5 \\
Identificación & 13 \\
Alimentos & 7 \\
Institucional & 4 \\
Custodia & 5 \\
Total & 335 \\
\hline
\end{tabular}

Fuente: elaboración autores

Tabla. 4: Período de enero - septiembre del 2013 (año actual)

\begin{tabular}{ll}
\hline Tipos de maltrato & $\mathrm{N}^{\circ}$ denuncias \\
\hline Físico & 54 \\
Psicológico & 42 \\
Sexual & 7 \\
Discriminación & 2 \\
Identificación & 5 \\
Alimentos & 3 \\
Institucional & 2 \\
Custodia & 5 \\
Problemas conductuales & 19 \\
Abandono & 9 \\
Total & 148 \\
Total de casos atendidos (2011-2013) & 483 \\
\hline
\end{tabular}

Fuente: Junta Cantonal de Protección de Derechos de Archidona.

Tabla. 5

Datos estadísticos sobre problemas que afectan a la seguridad ciudadana en relación a niños, niñas y adolescentes del Cantón Archidona- enero a julio del 2015

\begin{tabular}{lll}
\hline Problemas & $\mathbf{N}^{\circ}$ de denuncias & Porcentaje \\
\hline $\begin{array}{l}\text { Consumo de bebidas } \\
\text { alcohólicas }\end{array}$ & 23 & $18 \%$ \\
$\begin{array}{l}\text { Consumo de sustancias } \\
\text { estupefacientes y } \\
\text { psicotrópicas }\end{array}$ & 8 & $6 \%$ \\
$\begin{array}{l}\text { Callejizacion } \\
\begin{array}{l}\text { Abandono a niños, niñas y } \\
\text { adolescentes }\end{array}\end{array}$ & 25 & $9 \%$ \\
Embarazo en adolescentes & 5 & $19 \%$ \\
\hline
\end{tabular}




\begin{tabular}{lll}
\hline $\begin{array}{l}\text { Deserción escolar } \\
\begin{array}{l}\text { Adolescentes infractores } \\
\text { (hurto) }\end{array}\end{array}$ & 3 & $2 \%$ \\
$\begin{array}{l}\text { Maltrato a niños, niñas y } \\
\text { adolescentes }\end{array}$ & 50 & $4 \%$ \\
Total & 131 & $38 \%$ \\
\hline
\end{tabular}

Fuente: Elaboración autores.

Del total de casos atendidos y registrados del 2011 al 2013, se evidencia que el maltrato físico está en primer lugar con 269 denuncias; el maltrato psicológico se ubica en segundo lugar con 113 denuncias; denuncias de connotaciones sexual son 22 casos y en el año 2013 se receptan 19 denuncias por problemas conductuales. Durante el año 2015, llama la atención el abandono a niños, niñas y adolescentes con 25 denuncias y el consumo de bebidas alcohólicas con 23 casos. Resaltando que el maltrato en general, es decir, el maltrato físico, psicológico y sexual sigue en primer lugar con 50 denuncias receptadas y atendidas.

Correlacionando los datos registrados por la Junta Cantonal de Protección de Derechos y los datos recogidos en la encuesta cantonal, demuestran que el maltrato es cotidiano, y como muestran las estadísticas a nivel nacional y mundial, las mujeres, los niños, las niñas, los indígenas y los de piel distinta sufren los peores castigos y malos tratos.

En nuestro medio entra en juego el tema étnico, pero no por ello se puede concebir las "costumbres" como formas adecuadas de educación y crianza. Las encuestas a nivel nacional, muestran que el maltrato no es un "método exclusivo" de los indígenas o de los pobres, el maltrato $^{2}$ está presente en todos los estratos sociales, a nivel urbano y rural. Es decir, el maltrato y violencia a niños, niñas y adolescentes es una homogeneidad que alarma (. En la encuesta del ODNA desde el año 2000 hasta el 2010, se pueden apreciar los siguientes datos:

\footnotetext{
${ }^{2}$ El art. 67 del Código de la Niñez y Adolescencia dice: Se entiende por maltrato toda conducta, de acción u omisión, que provoque o pueda provocar daño a la integridad o salud física, psicológica o sexual de un niño, niña o adolescente, por parte de cualquier persona, incluidos sus progenitores, otros parientes, educadores y personas a cargo de su cuidado; cualesquiera sean el medio utilizado para el efecto, sus consecuencias y el tiempo necesario para la recuperación de la víctima. Se incluye en esta calificación el trato negligente o descuido grave o reiterado en el cumplimiento de las obligaciones para con los niños, niñas y adolescentes, relativas a la prestación de alimentos, alimentación, atención médica, educación o cuidados diarios; y su utilización en la mendicidad.
} 


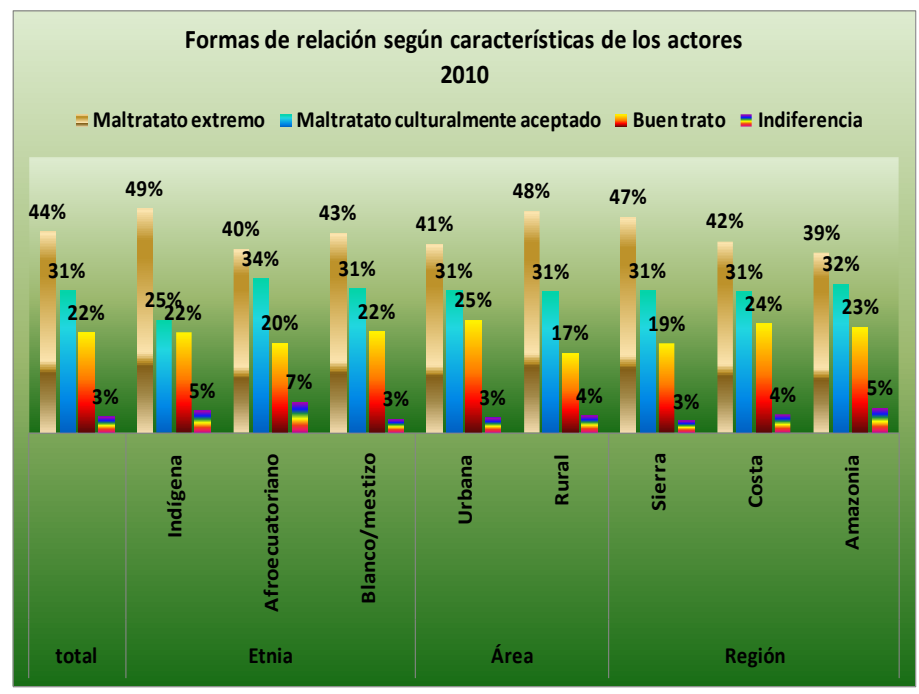

Fuente: elaboración autores.

En este gráfico se puede apreciar que en nuestro medio entra en juego el tema étnico, pero no por ello se puede concebir las "costumbres" como formas adecuadas de educación y crianza. Las encuestas a nivel nacional, muestran que el maltrato no es un "método exclusivo" de los indígenas o de los pobres, el maltrato ${ }^{3}$ está presente en todos los estratos sociales, a nivel urbano y rural. Es decir, el maltrato y violencia a niños, niñas y adolescentes es una homogeneidad que asusta.

A estas alturas de la historia, sorprende que los adultos no hayamos comprendido que la violencia no reeduca ni cambia las "conductas" socialmente dañinas. A estas formas de relación siempre subyace la actitud adulto céntrica de mantener el dominio y reestablecer el principio de autoridad (Calle, 2012).

Si en la gráfica anterior se constata que, el maltrato extremo en el sector indígena, alcanza el 49\%, seguidamente el maltrato culturalmente aceptado alcanza su mayor porcentaje de $48 \%$ a nivel rural y, a nivel regional la sierra llega al $48 \%$ dentro de la categoría de maltrato extremo. Sin embargo resaltamos que el maltrato no puede ser abordado como un tema cultural, sino como "método" o práctica social, enmarcada en las lógicas de relaciones de poder ${ }^{4}$.

\footnotetext{
${ }^{3}$ El art. 67 del Código de la Niñez y Adolescencia dice: Se entiende por maltrato toda conducta, de acción u omisión, que provoque o pueda provocar daño a la integridad o salud física, psicológica o sexual de un niño, niña o adolescente, por parte de cualquier persona, incluidos sus progenitores, otros parientes, educadores y personas a cargo de su cuidado; cualesquiera sean el medio utilizado para el efecto, sus consecuencias y el tiempo necesario para la recuperación de la víctima. Se incluye en esta calificación el trato negligente o descuido grave o reiterado en el cumplimiento de las obligaciones para con los niños, niñas y adolescentes, relativas a la prestación de alimentos, alimentación, atención médica, educación o cuidados diarios; y su utilización en la mendicidad.

${ }^{4}$ Para Foucault (1993) el poder se construye y funciona a partir de otros poderes, de los efectos de estos, independientes del proceso económico. Las relaciones de poder se encuentran estrechamente ligadas a las familiares, sexuales, productivas; íntimamente engarzadas y desempeñando un papel de condicionante y condicionado. En el análisis del fenómeno del poder no se debe partir del centro y descender, sino más bien realizar un análisis de abajo hacia arriba, a partir de los "mecanismos infinitesimales", que poseen su propia historia, técnica y táctica, y observar cómo estos procedimientos han sido colonizados, utilizados, transformados, doblegados por formas de dominación global y mecanismos más generales.
} 


\section{Hacia una cultura del buen trato}

Si estos datos sorprenden, nos vemos en la obligación imperante de seguir promoviendo prácticas de buen trato para elevar el 22\% que alcanzamos hasta el 2010, pero sobre todo para no alimentar esa postura de indiferencia que muchos padres y educadores están adoptando, como respuesta a la impotencia. Pero, ¿cuáles son las mejores prácticas o formas de relación adecuadas, que se pueden decir de buen trato? Este planteamiento fue hecho a los padres de familia y a sus hijos en el cantón Archidona y se obtuvieron algunos resultados.

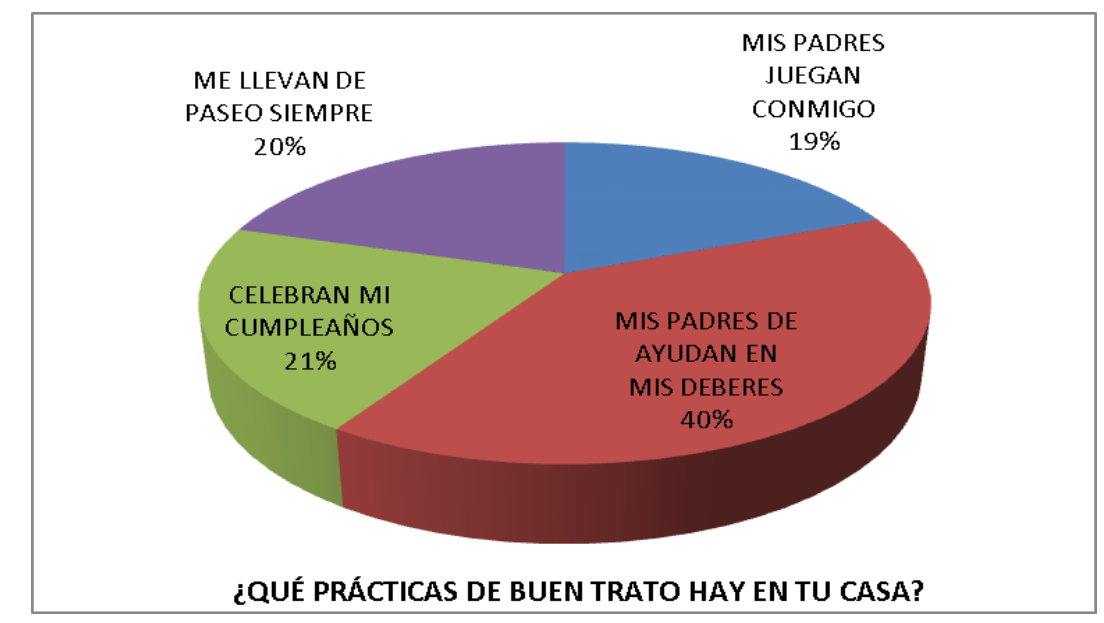

Fuente: elaboración autores.

Como se puede observar los niños, niñas y adolescentes, afirmaron que sus padres les ayudan en sus deberes o tareas de la escuela en un $40 \%$, las demás respuestas difieren en el $1 \%$. Estas respuestas son muy parecidas a la de los padres, porque ellos dicen ayudar en los deberes, $44 \%$ y que juegan con sus hijos un $20 \%$.

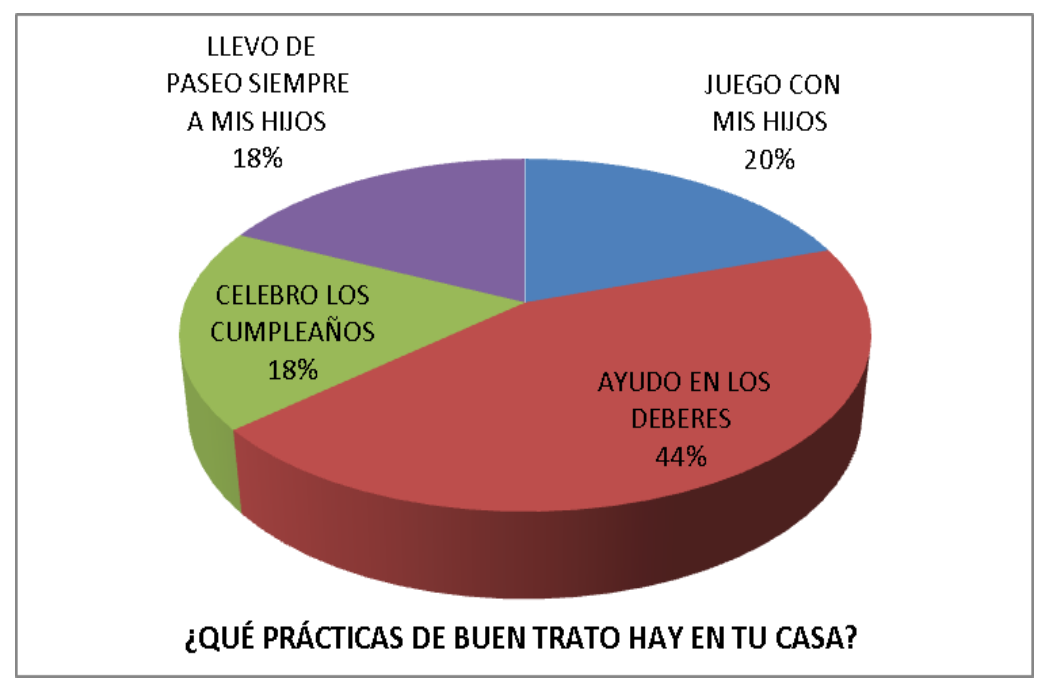

Fuente: elaboración autores. 
En un ambiente de naturalización de la violencia (, dominado por el adulto centrismo, resultó muy difícil obtener evidencias de buen trato. De hecho, los niños, niñas y jóvenes relacionan el buen trato con las esporádicas muestras de afecto de sus padres y generalmente piensan en los regalos que han recibido en las celebraciones de cumpleaños. Otro hecho positivo que ellos comentan es que, desde hace tres años reciben apoyo de la Fundación Mariana de Jesús. Este apoyo consiste en chequeos médicos, encuentros de formación y viajes a otros lugares, campamentos vacacionales y en muchas ocasiones reciben víveres para complementar la canasta familiar. Los factores que posibilitarían mejores relaciones entre adultos y niños y, sobre todo garantizarían una mejor comunicación para escuchar, sentir y hacer desde la diversidad, son las muestras de afecto.

\section{Conclusiones}

Si el significado de "tener derechos" es satisfacer necesidades, desarrollar habilidades y alcanzar metas, determinamos que lejos de cumplir esta premisa, los niños, niñas y adolescentes del cantón Archidona, en su mayoría del sector rural e indígena, están relegados a un segundo plano, prueba de ello es el déficit de servicios básicos como el agua potable. Los factores que contribuyen para que niños en su primera infancia padezcan desnutrición crónica en este cantón son principalmente estructurales, como la pobreza reflejada en recursos económicos, características de la familia, acceso a servicios higiénicos y educación, entre otros ${ }^{5}$.

Las relaciones afectivas entre adultos y niños, niñas y adolescentes en el cantón Archidona, se asemejan a las cifras de nivel nacional. Aunque los niños, niñas, adolescentes y sus padres califican su relación como muy buena y buena respectivamente, a la hora de "corregir conductas", no dudan en afirmar que aplican diversos castigos. Esta contradicción, se debe a que la violencia es un hecho que cruza toda la historia y, se lo debe entender cómo relación de poder interétnico (Baumann, G. 2001; Castañeda, C. 1999; Fuentes, B. 1997). Considerada la violencia como un tema y problema que oscila entre la tolerancia e intolerancia social, se puede observar su profunda inserción en todas las actividades del ser humano ya sea en forma explícita o solapada, pues el arte, la política, las relaciones, interindividuales, las colectivas, la tecnología, etc., también están inmiscuidas en su práctica real, en su afirmación, difusión y consecuencias (Adoum, 2005; Garcés, 2006).

Según las cifras arriba graficadas y analizadas, podemos admirar que la violencia y su práctica habitual la han naturalizado, provocando una especie de encadenamiento de las violencias, desde la violencia de género, étnica, política, etc. Los esfuerzos por reducirla han llevado al Ecuador a ejecutar varias políticas poco efectivas, ya que por un lado se persigue y castiga a los que provocan daño ejerciendo violencia, no se atacan a los factores que lo alimentan y protegen como las condiciones estructurales de vida de los pobladores. Este esfuerzo en el contexto estudiado, se matiza con ciertas tradiciones y formas de vida consideradas "propias" de la cultura kichwa. Al respecto se puede afirmar que, en algunos casos, la persistencia de

\footnotetext{
${ }^{5}$ Estos factores fueron los más importantes al momento que se realizó la investigación en el Cantón Archidona, en el año 2012, con el apoyo de dos estudiantes de la fundación CIMAS. Por su contenido, este trabajo cuestiona las cifras oficiales sobre desnutrición, ya que la metodología aplicada fue in situ, directamente con las familias que habitan en este lugar.
} 
costumbres y pensamientos puede verse como resultado del desigual acceso a los bienes de la modernidad.

En un ámbito marcado por relaciones poco afectivas, fomentar una desestructuración de lo social, resquebrajando los lazos culturales (Aguilar, 2018). El estado, la sociedad y la familia, deben garantizar, dentro de sus ámbitos, el cumplimiento efectivo de los derechos de niños, niñas y jóvenes. La protección integral debe aterrizar y ser muy práctica, al Estado le compete garantizar el acceso a una educación, salud y otros servicios de calidad ${ }^{6}$. Del mismo modo las entidades del sistema de Protección, particularmente los de Justicia, deben revisar y adaptar su legislación a las lógicas locales. La familia por su parte, como instancia social diversa, configurada por la globalización y otros factores, incluso debe evitar convertirse en reproductora de violencias, debe volcar su mirada al punto de vista de los actores y actoras sociales.

\section{Bibliografía}

Adoum, J. 2005. Ensayo. Obras (in) completas. Quito: Casa de la Cultura Ecuatoriana Benjamín Carrión.

Aguilar, R, W. 2018. Estudio etnográfico sobre violencias y drogas en Quito. Revista Chilena de Antropología, (38), 369-383. doi:10.5354/0719-1472.2019.51365

Aguilar, F y Tingo, F. 2016. Violencia y protección internacional: relatos de niños y niñas en la frontera norte del Ecuador. Universitas, Revista de Ciencias Sociales y Humanas, núm. 25, pp. 23-45.

Auyero, J y Berti M. 2013. La violencia en los márgenes. Buenos Aires: Katz.

Baumann, G. 2001. El enigma multicultural. Un replanteamiento de las identidades nacionales, étnicas y religiosas. Barcelona: Ediciones Paidós.

Berthelot, J. 2003. La construcción de la Sociología. Buenos Aires: Editorial Nueva Visión.

Böll, V., Conejo, M., Costales, X., Lozano, A., Schlegl, A., Tocagón, L., Wisum, C., 1997. Identidades indígenas en las ciudades. Quito: Fundación Hanns Seidel.

Bourdieu, P. 2000. La dominación masculina. Barcelona: Anagrama.

Bourdieu, P., Loic W. 2005. Una invitación a la sociología reflexiva. Buenos Aires: Siglo XXI.

Bourgois, P. 2002. El poder de la violencia en la guerra y la paz. Apuntes de Investigación, VI (8): 73-98.

\footnotetext{
${ }^{6}$ La atención de calidad es aquella que se aproxima al "ser individual y colectivo" para encontrar en la condición biológica, psicológica, espiritual; las capacidades y debilidades que le permiten construir un proyecto de vida individual y/o colectiva para una vida digna y feliz.
} 
Bourgois, P. 2010. En busca de Respeto, vendiendo crack en Harlem. Buenos Aires: Siglo Veintiuno.

Calle, H. (coomp). 2012. Violencia y Maltrato en niños, niñas y adolescentes. Investigación, Prácticas Sociales Excluyentes y Respuesta Institucional.

Castañeda, C. 1999. La rueda del tiempo: Los chamanes del Antiguo México y sus pensamientos acerca de la Vida, la Muerte y el Universo. Barcelona: Gaia Ediciones.

Código de la Niñez y Adolescencia. 2011. Quito: Consejo Nacional de la Niñez y Adolescencia.

Constitución del Ecuador. 2008. Quito: Publicación oficial de la Asamblea Nacional.

Del Olmo, R. 2000. “Ciudades duras y violencia urbana”. En Revista Nueva Sociedad, No. 167: 74-86. Buenos Aires: FES.

Ferrándiz, M. y Feixa, C. 2004. Una mirada antropológica sobre las violencias Alteridades vol. 14, núm. 27, pp. 159-174. Universidad Autónoma Metropolitana Unidad Iztapalapa Distrito Federal, México.

Foucault, M. 1993. Vigilar y castigar. Nacimiento de la prisión. México: Siglo veintiuno editores.

Fuentes, B. 1997. Huaomoni, Huarani, Cowudi. Una aproximación a los Huaorani en la práctica política, multi-étnica ecuatoriana. Colección Pueblos del Ecuador 14. Quito: Ediciones Abya Yala.

García, N. 2001. Culturas Hibridas. Estrategias para entrar y salir de la modernidad. Argentina: Ediciones Paidós.

Garcés, A. 2006. Relaciones de Género en la Amazonía Ecuatoriana. Estudio de caso en comunidades indígenas Achuar, Shuar y Kichua. Quito: Ediciones Abya Yala, CEDIME y ded.

Palacios, J., Marchesi, A., Coll, C. 2002. Desarrollo Psicológico y Educación. 1. Psicología Evolutiva. Madrid: Alianza Editorial.

Sánchez, P. 1989. “QQué es un niño? Notas para una crítica de la razón asistencial”. Ecuador Debate, no. 19 (diciembre): 141-158.

Sánchez, P, José. 2004. Orfandades infantiles y adolescentes. Para una sociología de la infancia. Quito: Abya Yala.

Sánchez, P, José. 2007. Una “devastación de la inteligencia”. Crisis y crítica de las Ciencias Sociales. Quito: Abya Yala.

Sémelin, J. 1983. Para escapar de la violencia. París: Edición de los trabajadores. 